'Unidad de Cuidados Intensivos HUAP Hospital de Urgencia Asistencia Pública (HUAP). Santiago, Chile.

${ }^{2}$ Universidad Nacional Andrés Bello. Santiago, Chile. ${ }^{\text {a}}$ Enfermero.

Conflictos de interés: todos los autores declaran no tener conflictos de interés. Trabajo no recibió financiamiento.

Recibido el de 29 abril de 2018, aceptado el 22 de junio de 2018.

Correspondencia a: Vinko Tomicic

Unidad de Cuidados Intensivos Hospital de Urgencias Asistencia

Pública. Portugal 125. Quinto piso. Santiago, Chile. Teléfono: 25681085.

Mesa Central: 25681100. vtomicic@gmail.com

\section{Adsorción extracorpórea de citoquinas en el tratamiento del shock séptico refractario. Casos clínicos}

\author{
MARIO RATHKAMP ${ }^{1}$, VINKO TOMICIC ${ }^{1,2}$, \\ JUAN CORNEJO ${ }^{1,2}$, PABLO CRUZ ${ }^{1,2, a}$
}

\section{Extracorporeal cytokine hemadsorption for the treatment of refractory septic shock. Report of two cases}

\begin{abstract}
If Septic shock (SS) evolves to refractory SS, mortality could reach 90\%, despite giving an optimal treatment. Nowadays, extracorporeal devices which adsorb inflammatory cytokines are available, reducing the systemic inflammatory response syndrome. These devices can be used with continuous renal replacement therapy or conventional hemodialysis. We report two diabetic females aged 50 and 58 years, who underwent a total colectomy and amputation of diabetic foot and who developed a SS with high requirements of vasoactive drugs (norepinephrine and adrenaline) to maintain a mean arterial pressure about $60 \mathrm{mmHg}$. Both were subjected to hemodialysis, connected to a cytokine hemadsorption device. The most important finding was the progressive reduction of vasopressor doses, effect that was observed nine hours after the beginning of the hemadsorption and lasted until its removal at 26 hours. Both patients survived.
\end{abstract}

(Rev Med Chile 2018; 146: 796-801)

Key words: Catecholamines; Cytokines; Hemadsorption; Shock, Septic.
L a sepsis es una de las causas más frecuentes de muerte en las unidades de cuidados intensivos (UCI) y si ésta progresa a shock séptico (SS) refractario, la mortalidad puede llegar a ser $\geq 90 \%$, a pesar de administrar tratamiento médico óptimo ${ }^{1,2}$. La principal causa de muerte son la disfunción orgánica múltiple (DOM) o el shock progresivo con hipotensión refractaria.

La inflamación es la respuesta normal que desarrolla el organismo frente a una noxa y se caracteriza por atraer polimorfonucleares, monocitos, oxígeno y nutrientes a la zona afectada, para asistir en el proceso de reparación y cicatrización ${ }^{3}$. Desafortunadamente, el sistema inmune frecuentemente sobre-reacciona frente a la condición desencadenante (sepsis, trauma, quemaduras, SDRA) conduciendo a una liberación masiva de mediadores inflamatorios, lo cual se ha deno- minado "tormenta citoquínica". Las citoquinas constituyen un centenar de pequeñas proteínas que regulan la respuesta inmune donde están incluidas la familia de las interleuquinas, interferón, factor de necrosis tumoral, entre otros ${ }^{4}$.

La tormenta citoquínica agrede al organismo de tal forma, que conduce a un proceso inflamatorio generalizado, originalmente denominado síndrome de respuesta inflamatoria sistémica (SRIS). Este proceso se caracteriza por una cascada de eventos que llevan a la muerte celular, disfunciones orgánicas y finalmente a la muerte. Hoy se sabe que el incremento de los niveles de citoquinas se correlaciona con la gravedad y la mortalidad de la sepsis ${ }^{5,6}$.

La modulación de la cascada de citoquinas posee el potencial para mitigar la magnitud del SIRS, reduciendo así la gravedad de la enferme- 
dad, contribuyendo a que los pacientes puedan recuperarse y sobrevivir. Hasta hace poco, la hemofiltración de alto volumen de inicio $\operatorname{precoz}^{7,8} \mathrm{y}$ la administración de corticoides ${ }^{9}$ eran las únicas herramientas clínicas que podían limitar la progresión de la cascada de citoquinas; el primero, al filtrar algunas citoquinas proinflamatorias; la segunda, al estimular la síntesis de I-kB y su unión con la cadena p65, impidiendo la formación del NF-kB activo, pero ambas indicaciones cuentan actualmente con evidencia débil ${ }^{10,11}$.

Recientemente, se han desarrollado sistemas extracorpóreos capaces de adsorber eficientemente citoquinas de la circulación y así disminuir la respuesta inflamatoria ${ }^{12,13}$. Estos dispositivos contienen esferas de polímero poroso biocompatible capaces de retirar citoquinas de la circulación y además de otros elementos que pueden contribuir con la respuesta inflamatoria: toxinas bacterianas, bilirrubina, mioglobina, complemento activado y mediadores capaces de promover y amplificar el SRIS. Estos dispositivos son compatibles con la hemodiálisis (HMD), reemplazo renal continuo y con los sistemas de soporte extracorpóreos de la oxigenación. Existen datos clínicos de pacientes con SS y cirugía de revascularización coronaria que han mostrado mejoría hemodinámica y reducción de la necesidad de vasopresores ${ }^{12,13}$. La dosis acumulativa de éstos últimos se correlaciona con la ocurrencia de insuficiencia circulatoria, acidosis metabólica, falla renal y con la mortalidad en $\mathrm{UCI}^{14}$

$\mathrm{Al}$ enfrentarnos frecuentemente con pacientes jóvenes con SS refractario a la terapia convencional óptima y al uso de terapias de reemplazo renal con cuadros clínicos potencialmente reversibles, decidimos incorporar un dispositivo de hemoadsorción en dos pacientes quirúrgicos graves que se hospitalizaron en nuestra unidad.

\section{Material y Métodos}

Se trataron dos pacientes, en la UCI-HUAP, que evolucionaron con SS refractario, que, a pesar de la reanimación con fluidos, uso de vasopresores y corticoides, mantuvieron el lactato mayor a 2,9 $\mathrm{mmol} / \mathrm{l}$, necesidad de noradrenalina (NA) $\geq 0,3$ $\mu \mathrm{g} / \mathrm{kg} / \mathrm{min}$, o la necesidad de asociar adrenalina (AD) para mantener la presión arterial media $(\mathrm{PAM}) \geq 60 \mathrm{mmHg}$.
Se utilizó un dispositivo denominado CytoSorb $^{\circledR}$ (CytoSorbents Corporation, Monmouth Junction, NJ, USA) biocompatible, compuesto por polímeros porosos que logran la unión permanente con moléculas entre 5-60 kDa, tales como: TNF- $\alpha$, IL-1 $\beta$, IL-6 y IL-10. Este equipo se anexó a una máquina de HMD convencional, previo a un filtro de diálisis FX 100, que fue programada con QB de $150 \mathrm{ml} / \mathrm{min}$, QD $300 \mathrm{ml} / \mathrm{min}$, tasa de UF cero, que se mantuvo por $24 \mathrm{~h}$. Posteriormente se utilizó solo HMD intermitente extendida. Los pacientes consintieron para la publicación de sus datos manteniendo la confidencialidad.

\section{Resultados}

\section{Caso 1}

Mujer de 50 años, diabética, hipertensa y dislipidémica, que se presenta en el servicio de urgencia (SU) de HUAP con dolor abdominal difuso. Se describe taquicárdica, taquipneica y mal perfundida. Se hospitaliza y se realiza TAC de abdomen y pelvis que muestra importante íleo colónico sin signos de obstrucción mecánica, ni perforación de víscera hueca, que se interpreta como síndrome de Ogilvie. Se inicia tratamiento antibiótico con ceftriaxona y metronidazol. La paciente evoluciona en malas condiciones, requiriendo intubación orotraqueal, ventilación mecánica $(\mathrm{VM})$ y NA. Evaluada por equipo quirúrgico y en presencia de abdomen agudo se decide ingresar a pabellón. En protocolo operatorio se describe gran cantidad de líquido purulento ( $\pm 400 \mathrm{cc}$ ), necrosis de todos los segmentos del colon, por lo que se realiza colectomía total e ileostomía terminal, aseo con laparostomía e instalación de Abthera.

Ingresa a UCI con diagnósticos de sepsis de foco abdominal, necrosis colónica de etiología no precisada, laparostomía y DOM. Se efectúa reanimación guiada por metas y soporte vital avanzado $^{10,15}$. Se inicia HMD extendida asociada a Cytosorb $16 \mathrm{~h}$ después del ingreso a UCI. La paciente se estabiliza hemodinámicamente a $9 \mathrm{~h}$ del inicio de la terapia y la $\mathrm{AD}$ pudo ser retirada a las 19 h, momento en que la NA se había disminuido desde 0,6 al ingreso a $0,12 \mu \mathrm{g} / \mathrm{kg} / \mathrm{min}$ (Figura 1). En forma paralela se observó mejoría de los parámetros metabólicos (Figura 2). Al $6^{\circ}$ día fue extubada exitosamente. Durante su estadía en UCI la paciente fue sometida a tres aseos qui- 


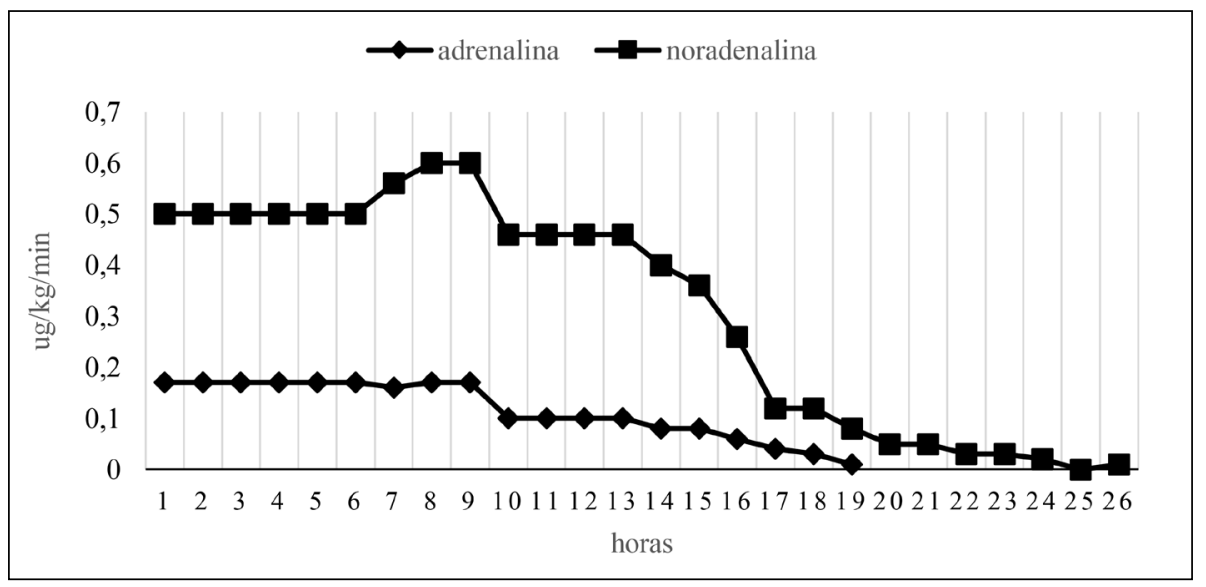

Figura 1. Dosis de NA y AD durante el shock inicial.

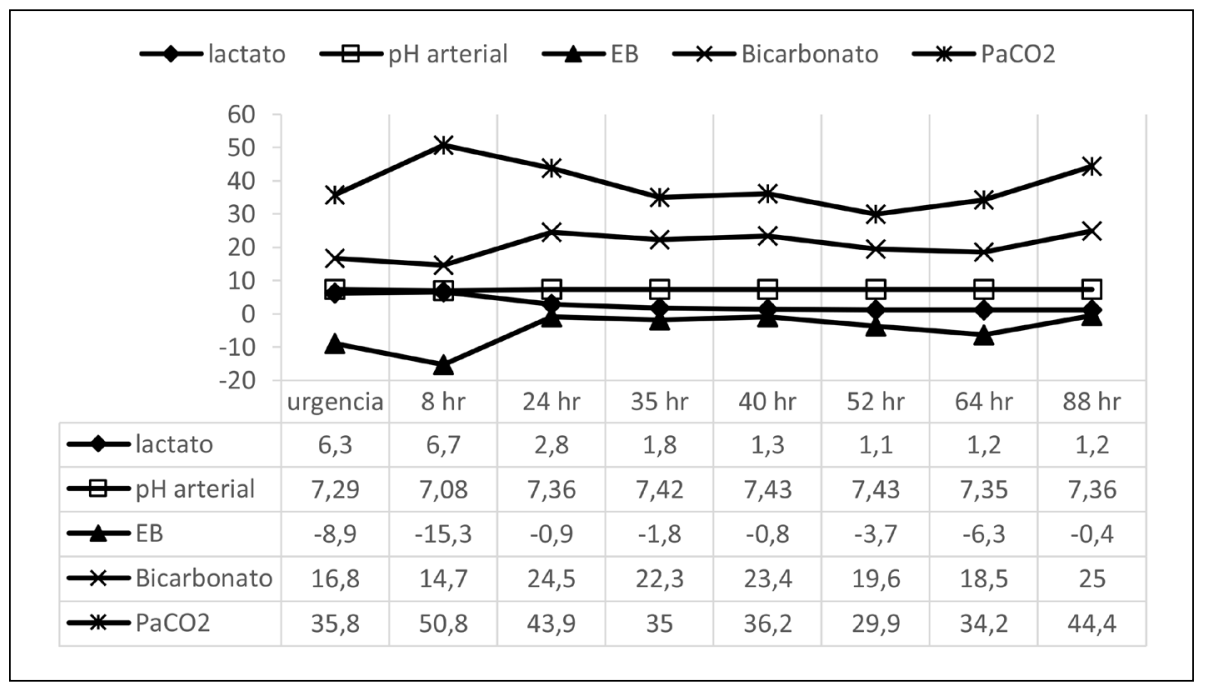

Figura 2. Parámetros metabólicos. Pre, durante y post $\mathrm{HMD}+$ hemoadsorción.

rúrgicos antes de cerrar la laparostomía. Durante toda su evolución, la diuresis se mantuvo entre 430 y $1.005 \mathrm{ml} /$ día, requiriendo HMD hasta el día 28 de hospitalización. Las variables demográficas, días de estada en VMI, UCI y UTI se describen en la Tabla 1.

\section{Caso 2}

Mujer de 48 años derivada por pie diabético complicado. Llegó a SU HUAP con dolor abdominal tipo cólico en hipocondrio derecho de dos días de evolución, asociado a ictericia sin fiebre. La ecotomografía abdominal descartó alteraciones en vesícula y vías biliares. Se inicia tratamiento antibiótico con ceftriaxona, clindamicina y ami- kacina. Doce horas después, la paciente ingresa a pabellón en malas condiciones generales, donde desarrolla taquicardia ventricular y PCR que fue reanimado. Se efectúa amputación infracondílea abierta. Ingresa a UCI intubada, en VM, taquicárdica, con $0,35 \mu \mathrm{g} / \mathrm{kg} / \mathrm{min}$ de NA, con presión arterial de 54/36 mmHg, acidótica, con síndrome de DOM (hemodinámico, renal, hematológico y hepático). Oligoanúrica e hiperkaliemica, se inicia HMD extendida asociada a Cytosorb $20 \mathrm{~h}$ después de su ingreso. La paciente se estabiliza hemodinámicamente a las $10 \mathrm{~h}$ de iniciada la terapia (Figura 3). La AD fue retirada a las $5 \mathrm{~h}$ de iniciado el procedimiento. Las variables demográficas, días de VM, UCI y UTI en la Tabla 1. 
Tabla 1. Características de los pacientes y su estadía en unidades críticas

\begin{tabular}{|lll|}
\hline Parámetros & Paciente $\mathbf{1}$ & Paciente $\mathbf{2}$ \\
\hline Edad & 50 años & 48 años \\
\hline Sexo & Femenino & Femenino \\
\hline APACHE II & 24 puntos & 23 puntos \\
\hline Inicio de HMD+Cytosorb & 16 h del ingreso & 20 h del ingreso \\
\hline Duración del shock & $26 \mathrm{~h}$ & $16 \mathrm{~h}$ \\
\hline VMI & 7 días & 6 días \\
\hline UCl & 10 días & 8 días \\
UTI & 14 días & 10 días \\
\hline
\end{tabular}

VMI: ventilación mecánica invasiva; UCI: unidad de cuidados intensivos; UTI: unidad de cuidados intermedios.

\section{Discusión}

El resultado más importante que muestran los casos descritos es que la asociación de hemodiálisis extendida con flujos bajos asociada con un dispositivo de hemoadsorción de citoquinas, fue segura y efectiva en el tratamiento del SS refractario en pacientes quirúrgicos, en los cuales el foco séptico fue removido precozmente. Existe literatura contradictoria respecto de los beneficios de las terapias de reemplazo renal ${ }^{7,8}$ y con el uso de hemoadsorción en pacientes con $\mathrm{SS}^{12}$. Sin embargo, en aquellos pacientes en que existe un grave disturbio del medio interno $(\mathrm{pH}<7,1$; bicarbonato $<10$ : exceso de base $<-10$ y lactato elevado), el uso de esta asociación ayudó a corregir dichas alteraciones contribuyendo a disminuir las dosis de NA y AD. La reducción pronta del soporte vasoconstrictor obtenida, probablemente atenuó la redistribución de flujo sanguíneo favoreciendo la circulación en los territorios esplácnico y renal, lo que quedó demostrado por la pronta recuperación metabólica (Figuras 2 y 4) y la recuperación parcial de la diuresis en ambos pacientes ${ }^{16,17}$. Lo anterior, podría reducir la magnitud de la translocación bacteriana desde el tubo digestivo y contribuir a mitigar el desarrollo de DOM, claves en la recuperación del $\mathrm{SS}^{18}$. Además, se postula que los beneficios observados con la hemoadsorción pueden deberse, en parte, a la protección de la función endotelial ${ }^{19}$.

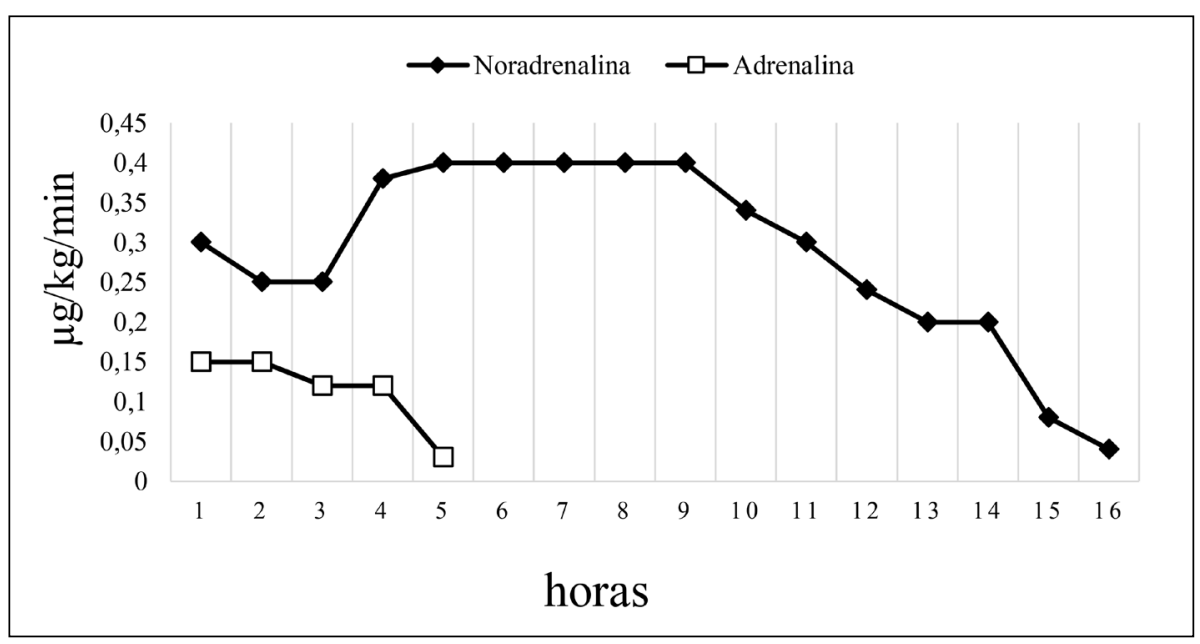

Figura 3. Dosis de NA y $A D$ durante el uso de HMD+hemoadsorción. 


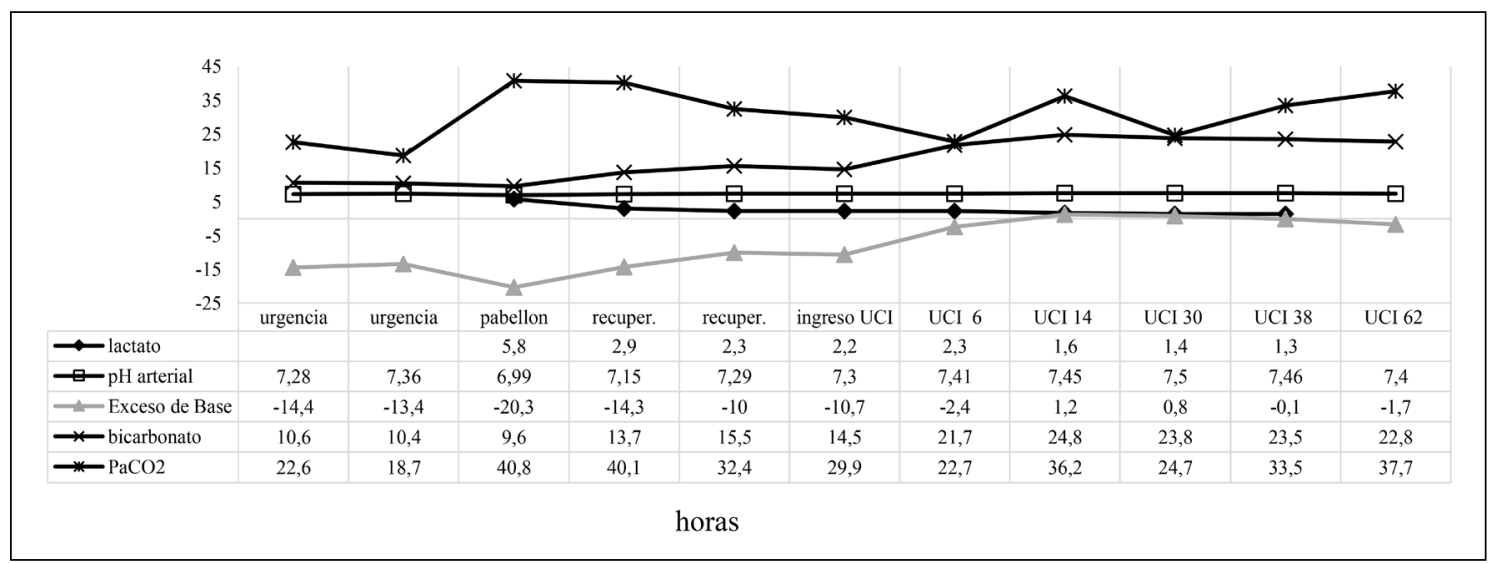

Figura 4. Evolución de los parámetros metabólicos pre, durante y post uso de HMD+hemoadsorción.

Kogelmann et al, reportaron que con la aplicación precoz de estos dispositivos de hemoadsorción (antes de 24 h), los resultados son mejores, tanto en la reducción del uso de vasopresores como en la sobrevida ${ }^{20}$.

Friesecke et al, observaron que la dosis de NA puede ser reducida significativamente a las $6 \mathrm{y}$ $12 \mathrm{~h}$ de iniciada la hemoadsorción: $-0,4 \mu \mathrm{g} / \mathrm{kg} / \mathrm{min}$ $(\mathrm{p}=0,03)$ y $-0,6 \mu \mathrm{g} / \mathrm{kg} / \mathrm{min}(\mathrm{p}=0,001)$ respectivamente, y la recuperación del SS se puede lograr en $65 \%$ de los pacientes. La mortalidad alcanzó el $45 \%$ con un SOFA de ingreso de $14,3 \pm 3$ puntos $^{21}$.

En nuestros pacientes fue posible retirar precozmente la $\mathrm{AD}$ y disminuir progresivamente la NA hasta suspenderla, siendo utilizada solo en algunas de las sesiones de HMD intermitente.

Estos antecedentes sugieren que el uso combinado de ambas técnicas, en el estadio precoz del SS, se puede considerar en aquellos casos en que las medidas rutinarias han fracasado en superar el shock refractario, atendiendo que su implementación no presenta mayor riesgo que la HMD convencional ${ }^{12-14}$. Es vital la remoción precoz del foco séptico y el uso de antibióticos apropiados. Este dispositivo si bien promisorio, posee un costo elevado y aún falta evidencia consistente sobre su impacto en la mortalidad.

\section{Referencias}

1. Engel C, Brunkhorst FM, Bone HG, Brunkhorst R, Gerlach H, Grond S, et al. Epidemiology of sepsis in Germany: results from a national prospective multicen- ter study. Intensive Care Med 2007; 33: 606-18.

2. Kumar G, Kumar N, Taneja A, Kaleekal T, Tarima S, McGinley E, et al. Nationwide trends of severe sepsis in the 21st century (2000-2007). Chest 2011; 140: 1223-31.

3. Tomicic V, Guerrero J. Endotelio y sepsis. Medicina Intensiva 2005; 29(3): 142-50.

4. Clark IA. The advent of the cytokine storm. Immunology and Cell Biology 2007; 85: 271-3.

5. Oda S, Hirasawa H, Shiga H, Nakanishi K, Matsuda K, Nakamua M. Sequential measurement of IL-6 blood levels in patients with systemic inflammatory response syndrome (SIRS)/sepsis. Cytokine 2005; 29: 169-75.

6. Kellum JA, Kong L, Fink MP, Weissfeld LA, Yealy DM, Pinsky MR, et al. Understanding the inflammatory cytokine response in pneumonia and sepsis: results of the Genetic and Inflammatory Markers of Sepsis (GenIMS) Study. Arch Intern Med 2007; 167: 1655-63.

7. Honore PM, Jamez J, Wauthier M, Lee PA, Dugernier T, Pirenne B, et al. Prospective evaluation of short-term, high-volume isovolemic hemofiltration on the hemodynamic course and outcome in patients with intractable circulatory failure resulting from septic shock. Crit Care Med 2000; 28: 3581-7.

8. Payen D, Mateo J, Cavaillon JM, Fraisse F, Floriot C, Vicaut E. Impact of continuous venovenous hemofiltration on organ failure during the early phase of severe sepsis: a randomized controlled trial. Crit Care Med 2009; 37: 803-10.

9. Annane D, Bellissant E, Bollaert PE, Briegel J, Confalonieri M, De Gaudio R, et al. Corticosteroids in the treatment of severe sepsis and septic shock in adults: a systematic review. JAMA 2009; 301: 2362-75.

10. Rhodes A, Evans LE, Alhazzani W, Levy MM, Antonelli M, Ferrer R, et al. Campaña para sobrevivir a la sepsis: 
recomendaciones internacionales para el tratamiento de la sepsis y el choque septicémico. Critical Care Medicine 2017; 45 (3): 486-567.

11. Borthwick EMJ, Hill CJ, Rabindranath KS, Maxwell AP, McAuley DF, Blackwood B. High-volume haemofiltration for sepsis in adults. The Cochrane Collaboration. Published by John Wiley \& Sons, Ltd. Copyright, 2017.

12. Schädler D, Pausch C, Heise D, Meier-Hellmann A, Brederlau J, Weiler N, et al. The effect of a novel extracorporeal cytokine hemoadsorption device on IL-6 elimination in septic patients: A randomized controlled trial. PLoS One 2017; 12 (10): e0187015.

13.- Bernardi MH, Rinoes H, Dragosits K, Ristl R, Hoffelner F, Opfermann P, et al. Effect of hemoadsorption during cardiopulmonary bypass surgery - a blinded, randomized, controlled pilot study using a novel adsorbent. Critical Care 2016; 20: 96.

14.- Dünser MW, Ruokonen E, Pettilä V, Ulmer H, Torgersen C, Schmittinger CA, et al. Association of arterial blood pressure and vasopressor load with septic shock mortality: a post hoc analysis of a multicenter trial. Crit Care 2009; 13: R181.

15.- Rivers E, Nguyen B, Havstad S, Ressler J, Muzzin A, Knoblich B, et al. Early Goal-Directed Therapy Collaborative Group. Early goal-directed therapy in the treatment of severe sepsis and septic shock N Engl J Med 2001; 345 (19): 1368-77.

16. Hosgood SA, Moore T, Kleverlaan T, Adams T, Nicholson ML. Haemoadsorption reduces the inflammatory response and improves blood flow during ex vivo renal perfusion in an experimental model. J Transl Med 2017; 15: 216.

17. Nacul FE, Guia IL, Lessa MA, Tibiriçá E. The effects of vasoactive drugs on intestinal functional capillary density in endotoxemic rats: intravital video-microscopy analysis. Anesth Analg 2010; 110 (2): 547-54.

18. Hernández G, Tomicic V. Effect of catecholamines on splanchnic perfusion in sepsis. Rev Med Chile 1999; 127 (6): 719-27.

19.- David S, Thamm K, Schmidt BMW, Falk CS, Kielstein JT. Effect of extracorporeal cytokine removal on vascular barrier function in a septic shock patient. Journal of Intensive Care 2017; 5: 12-7.

20.- Kogelmann K, Jarczak D, Scheller M, Drüner M. Hemoadsorption by CytoSorb in septic patients: a case series. Critical Care 2017; 21: 74.

21.- Friesecke S, Stecher S-S, Gross S, Felix SB, Nierhaus A. Extracorporeal cytokine elimination as rescue therapy in refractory septic shock: a prospective single-center study. J Artif Organs 2017; 20 (3): 252. 